\title{
Brasil Business Model - Considerações Sobre o Conceito
}

\author{
Recebido em 2013.08.16. Aprovado em 2014.10.10 \\ Avaliado pelo sistema double blind review
}

\author{
Paulo Cesar Lopes Pereira \\ prof.pc@ufri.br \\ Universidade Federal do Rio de Janeiro-RJ-Brasil
}

\section{Resumo}

O artigo apresenta criticamente os diferentes conceitos aplicados à expressão Business Model (BM), sua genealogia e algumas consequências de sua utilização por praticantes e acadêmicos. $O$ trabalho foi elaborado a partir da análise do material publicado sobre o assunto, utilizando as bases de dados de artigos científicos disponíveis no Portal Periódicos da CAPES, EBSCO Host e Scielo, apresentando exemplos e contra-exemplos buscados na Internet. Nas considerações finais são apresentados os aspectos comuns na conceituação da expressão, que emergem da análise dos artigos selecionados, bem como o potencial de aplicabilidade na academia e nos mundo dos negócios, com destaque à importância que o conceito pode ter para os empreendedores.

\section{Palavras-Chave: Modelo de Negócios, Inovação, Empreendedorismo.}

\begin{abstract}
This paper presents and criticise the different concepts of business model, its genealogy and possible effects of its use by academics and practicioners. The method used was the search and analysis of articles about the subject avaible in Portal Periódicos CAPES, EBSCO Host and Scielo databases, illustred with examples and counterexamples found in Internet. In the "Considerações Finais" section are presented the common issues underpinning the different proposals for the concept and its Science and Business aplicability, stressing its importance for entrepreneurs.
\end{abstract}

Keywords: Business Model, Innovation, Entrepreneurship.

\section{Apresentação}

A partir dos anos 90 um termo passa a figurar intensamente na literatura de negócios, especialmente entre os praticantes - Business Model. Na primeira década do século atual o termo passou a frequentar a literatura especializada, em especial nas áreas de TI, Inovação e Estratégia, havendo hoje farto material, muitas vezes apresentando, se não contradições, ao menos grandes controvérsias sobre o significado e aplicabilidade acadêmica do conceito.

Este artigo pretende apresentar os conceitos concorrentes, a genealogia do termo, os progressos e considerações sobre as diferentes abordagens e seus desdobramentos na pesquisa acadêmica em negócios, por meio de um levantamento bibliográfico de textos em português, inglês ou francês, feito nas bases de dados existentes no portal Periódicos Capes, na Business Premier da EBSCOhost e na Scielo. O método empregado para seleção dos artigos foi o denominado bola de neve, iniciado a partir das referências encontradas na obra de Chesbrough (2003), especificamente no capítulo "Business Model". O critério de 


\section{Brasil Business Model - Considerações Sobre o Conceito}

seleção foi a presença ao longo do texto de questões relativas à conceituação, gênese e desenvolvimento de aplicações da expressão Business Model, Modelo de Negócios ou Modèle Économique.

O trabalho está estruturado em 6 seções: esta Apresentação, uma Introdução, a Genealogia da Expressão, $\mathrm{O}$ Conceito de BM e as Considerações Finais.

\section{Introdução}

Modelo de Negócios, ou mais comumente escrito na sua forma original Business Model (BM) é uma expressão que vem se tornando habitual tanto no mundo leigo quanto acadêmicoi. Inicialmente são os praticantes (DEMIL et al, 2004) que demonstram maior interesse no assunto e a disseminação e discussão da noçãoil e ganha impulso a partir dos anos 90 (MAGRETTA, 2002).

Segundo Ostenwalder e Pigneur (2002) a importância do conceito decorreria da possibilidade que 0 constructo ofereceria para:

1. Entender os elementos relevantes em uma área de conhecimento específica;

2. Comunicar e disseminar entre os grupos de interesse (stakeholders) 0 entendimento [que a organização] tem sobre um determinado negócio;

3. Facilitar o processo de mudança (em sentido amplo, associado a um negócio em particular);

4. Identificar as medidas de desempenho relevantes para o desenvovimento de um negócioiii;

5. Simular e aprender sobre um determinado negócio (Como será visto posteriormente, esta é última é uma função de um conceito diferente, associado ao BM - seria o business modelling)

Para Chesbrough (2003) BM seria um constructo que ligaria as decisões técnicas (relativas à tecnologia) aos resultados econômicos por meio da conversão do valor potencial da tecnologia em valor econômico. Ele afirma que um BM bem elaborado sobre uma tecnologia não tão boa pode ter resultados empresariais melhores que uma tecnologia tecnicamente excelente, mas que não foi adequadamente desdobrada em um BM. Em outras palavras, o BM permitira maximizar os ganhos econômicos de uma dada tecnologia.

$\mathrm{Na}$ academia, segundo Arlotto et al (2011), o primeiro registro da noção de BM é encontrado ao longo do texto de Lang (1947)iv e aparece pela primeira vez no título em um artigo de Jones (1960) (OSTENWALDER et al, 2005). No início dos anos 70 Stanford (1972) publica um artigo onde o termo é utilizado no título, no entanto, trata-se em verdade de modelos de jogos de negócios e modelagem computacional de negócios e não na acepção que é utilizada aqui. De toda a forma, até o momento não há um consenso sobre o conceito de BM, mas vários desdobramentos em termos de proposições de relacionamentos com outros conceitos, taxonomias e aplicações têm aparecido na literatura especializada.

Possivelmente pela novidade do conceito, BM vem sendo debatido intensamente nos últimos anos. No Portal Periódico Capes, utilizando a expressão "business model", retornaram 980.943 referências avaliadas por pares ${ }^{v}$. Em uma pesquisa na base de dados Business Source Complete, disponível na EbscoHost, utilizando como termo de procura nos campos sumário, texto ou título Business Model, somente em artigos em revistas acadêmicas foram encontradas 1.488 entradas, sendo 43 nos 3 primeiros meses de 2013, 228 em 2012, 203

em 2011, 212 em 2010, 199 em 2009, 139 em 2008, 131 em 2007, 100 em 2006, 111 em 2005 e 116 em 2004, demonstrando o interesse que o termo tem despertado entre os acadêmicos. 
Essa dinâmica não se repete no Brasil. Uma pesquisa utilizando os termos "business model" "business models" e "modelo de negócio" e "modelos de negócios" empregadas no título, resumo ou texto de trabalhos produzidos em língua portuguesa, realizada em 13/04/2013 na base de dados Business Premier retornou somente 6, 17vi, 0 e 3 textos respectivamente. Desses, um era de 2009, quatro de 2010, quatro de 2011, 5 de 2012 e um até abril de 2013.

Outro levantamento feito junto à base Scielo, utilizando os mesmos critérios para título, assunto e resumo foram obtidos 2, 1, 1 e 1 artigos respectivamente. Observe-se, no entanto, apesar dos diferentes termos, foram encontrados somente 3 artigos diferentes. Em contrapartida, o mais antigo era de 2002, enquanto que na pesquisa citada anteriormente o mais antigo era de 2009.

Curiosamente, não obstante a abundância de trabalhos, na maioria dos artigos, os autores chamam a atenção que o conceito não é claramente explicitado.

$\mathrm{O}$ dissenso sobre o conceito de BM decorreria da novidade sua pouca estruturação em termos acadêmicos (DEMIL et al, 2004). Sua característica transdisciplinar, envolvendo ao menos as áreas de marketing, estratégia, organização e finanças, faz com que não exista um domínio do saber que conduza 0 desenvolvimento do conceito.

\section{Genealogia da Expressão}

A genealogia do termo, popularizado, como já dito, nos anos 90, durante a bolha das empresas ".com" (MAGRETTA, 2002, OSTENWALDER et al, 2005, ARLOTO et al, 2011), pode ser decorrente dos novos negócios, novas formas de cooperação, competição e de apropriação de valor que apareceram com as Tecnologias de Informação e Comunicação (TIC's).

Em uma época revolucionária, como explicar a um investidor como o empreendimento irá faturar com aquele produto intangível, cuja matéria prima era fundamentalmente conhecimento (como os motores de busca na Internet) e que eventualmente não cobravam do usuário pela utilização do produto? Como mostrar que muitas das funções habituais - produção, marketing, distribuição, principalmente - não eram separadas da forma clássica, industrial, dominante. Como explicar que o desenvolvimento e produção não eram baseados em cadeias, mas sim em redes? Os instrumentos habituais - um plano de negócios, entre eles - muitas vezes não eram suficientes para tornarem inteligível a proposta, condição de possibilidade para que 0 potencial investidor ao menos considerasse a proposição de financiamento.

Para Verstraete e Juisson (2007) o conceito de BM é a expressão do processo que os empreendedores realizam para convencer os detentores de recursos a participarem formalmente do empreendimento, tornando-se parte dos grupos de interesse (stakeholders) envolvidos no projeto. Estes autores chamam a atenção que o BM se presta a apresentar o projeto dentro de um contexto de convenções (Teoria das Convenções - TC), isto é, somente por meio da utilização das convenções existentes, ou criando novas, no caso de inovação, as quais necessariamente são aceitas entre os envolvidos no processo de cooptação de fornecedores de recursos, os detentores dos recursos necessários à implementação do negócio aceitarão a possibilidade de tornarem-se grupos de interesse, ou seja, um comportamento pautado pela TC seria a condição básica para que o inovador pudesse ser compreendido pelos detentores de recursos.

Jóia e Ferreira (2005) argumentam que dadas as características da maior parte das conceituações por eles pesquisadas e que envolvem de uma forma ou outra, como será visto adiante, uma nova composição de recursos para oferecer um novo produto ao mercado, seria, então, razoável supor que um dos precursores do conceito seria Schumpeter, visto ele ter discutido o efeito da combinação de recursos na geração de novos produtos, mercados e setores industriais. 


\section{Brasil Business Model - Considerações Sobre o Conceito}

\section{Conceito de BM}

Um conceito é uma representação mental geral e arbitrária de um objeto, seja ele concreto (a empresa) ou abstrato (o conjunto de decisões envolvidas na entrada, ou mudança de comportamento em um mercado), estabelecida no seio de uma comunidade de saberes (a Academia) em um momento determinado (CHARTRAND e De KONICK, 2009).

Paralelamente a isso, para aplicar essa representação abstrata no mundo observável é necessário defini-la, o que pode ser feito por meio da enunciação dos atributos essenciais e específicos relativos àquele conceito $\mathrm{e}$ que o individualizariam frente a outros conceitos (MARTINS, 1996). Os trabalhos que envolvem a conceituação do BM assumem as duas expressões - ora o representam por meio da explicação de sua essência, ou por meio da definição, ou seja, por suas característicasvii.

Em um trabalho de 2002, Pateli fez um levantamento de alguns artigos, relacionando a forma de apresentar 0 conceito:

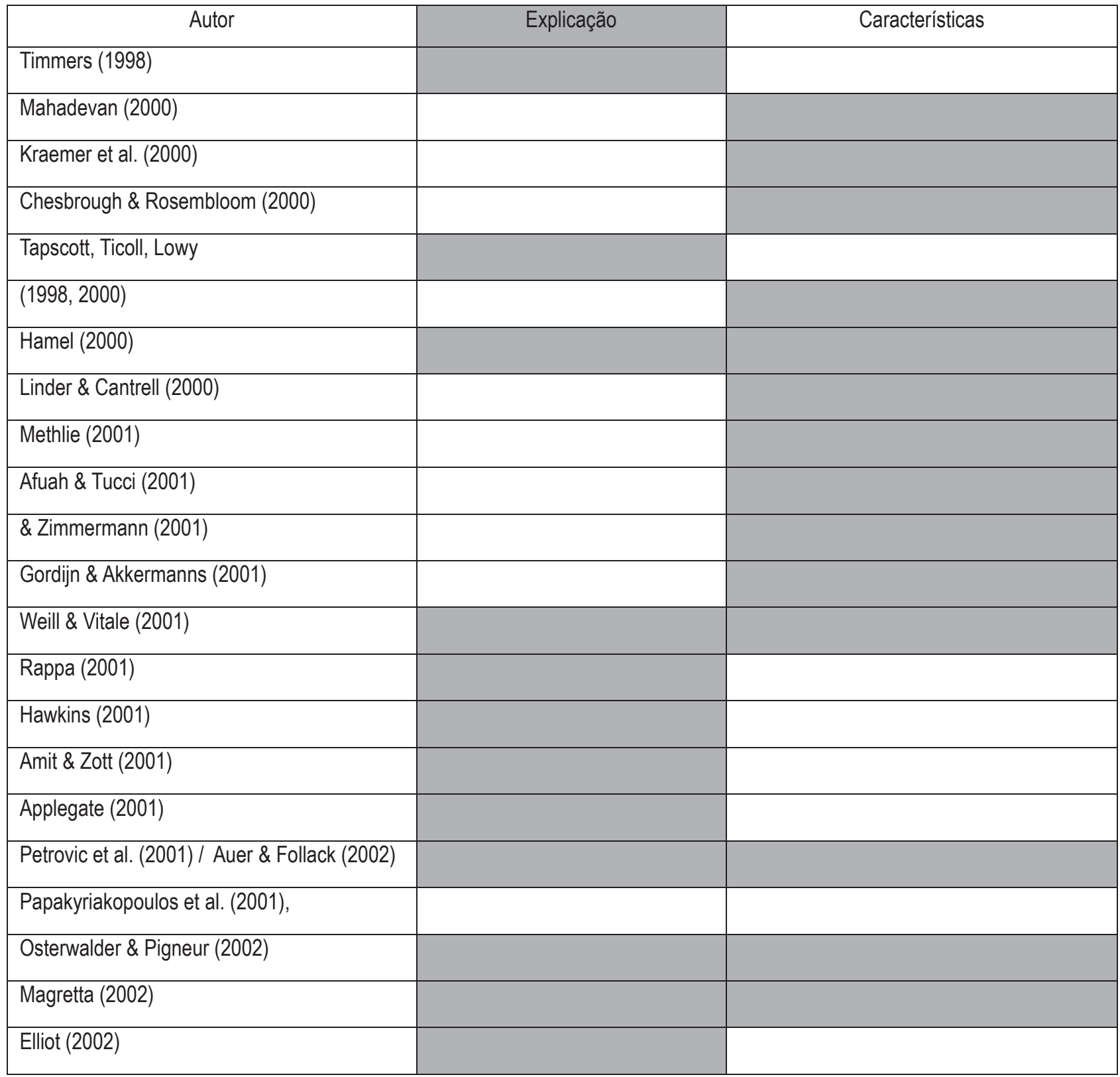

Quadro 1: Diferentes formas de apresentar o conceito de BM 
Fonte: adaptado de PATELI, 2002, Tabela1, p. 6

Em termos de desenvolvimento do conhecimento, a construção do conceito é um atributo dos pesquisadores (CHARTRAND e KONICK, 2009) e relevante porque, considerando Abbagnano (1970, apud MARTINS, 2005), o conceito cumpriria ao menos quatro funções sob o ponto de vista instrumental: 1) descreveria 0 objeto da experiência, permitindo seu reconhecimento ("isto se trata de um BM?"), 2) permitiria classificar o próprio conceito ("este é um BM inovador, ou não?), 3) organizaria os dados da experiência para serem estabelecidas conexões de natureza lógica ("um adequado BM permite converter o potencial valor da tecnologia em retornos financeiros"viii) 4) anteciparia a (possível ou provável) solução de um problema exatamente formulado ("o BM da empresa líder de um mercado define o padrão de competição nesse mercado, em situações de estabilidade competitiva"ix).

Em um artigo recente, Zoot et al (2010) apresentam um quadro com oito conceituações de BMx. Um ponto em comum em praticamente todas é que o conceito existe no âmbito da organização, isto é, relaciona-se com comportamentos e/ou decisões dela em um mercado. Dentre as conceituações apresentadas, três citam explicitamente firmas ou empresas. Teece (2010), um dos citados, inicia seu artigo com a seguinte afirmação: "Whenever a business enterprise is established, it either explicitly or implicitly employs a particular business model'(não destacado no original). Outros citados, Casadesus-Masanel e Ricart (2010), expandem a idéia, explicitando que BM é relativo à lógica da empresa.

Chesbrough e Rosembloon (2002) citados no mesmo artigo, apresentam o BM como sendo a descrição de como o valor potencial da tecnologias será parcialmente apropriado pela empresa. Neste caso, o objeto ao qual se refere o BM é representado pelas as decisões empresariais relativas à proposição de valor, identificação do segmento de mercado-alvo a estrutura da cadeia de valor necessária à produção da oferta e o mecanismo para a geração de retornos financeiros associados à troca, este último item é o que normalmente é mais citado na literatura.

Outras três conceituações citadas no mesmo artigo (TIMMERS, 1998, MORRIS et al, 2005, JONHSON et al, 2008) são apresentadas, tal como Chesbrough e Rosembloon (2002), por meio de seus constituintes, interligados e sempre atrelados a uma organização específica. Da mesma forma, Ethiraj et al (2000) definem BM como uma configuração única de elementos incluindo as metas organizacionais, estratégias, processos, tecnologias e estruturas concebidas para criar valor para os consumidores e, portanto, competir em um mercado.

Appelgate (2003, apud SEDON et al, 2004) identifica o BM por meio da descrição da oportunidade e estratégia adotada pela empresa em análise, a capabilidade, conceituada como recursos necessários para implementar a estratégia e a proposição de valor, especificamente para os investidores e outros grupos de interesse. Em um outro trabalho Demil et al (2004) seguem a questão da proposição de valor, em especial no que tange à apropriação de parte do valor criado, afirmando que o conceito é relativo exclusivamente às formas de remuneração da empresa.

Hedman e Kalling (2002) argumentam que o BM integraria os aspectos internos à firma que seriam transformados em recursos que, por seu turno, seriam aplicados em atividades dentro de uma determinada estrutur, os quais permitiriam a produção de produtos a serem oferecidos a um determinado mercado consumidor. Esse conjunto integrado permitiria certo grau de flexibilidade, i.é, a organização teria como variar a oferta, desde que não necessitasse de novas atividades ou competências ${ }^{x i}$, caso contrário desenvolveria um novo BM. Vale observar que a perspectiva dos autores sobre BM é a de projeto de processos - o BM seria uma ferramenta para mapear processos.

Além destes, pode-se recuperar uma conceituação dada por Osterwalder e outros (2005):

"A business model is a conceptual tool that contains a set of elements and their relationships and allows expressing the business logic of a specific firm. It is a description of the value a company offers to one or several segments of customers and of the architecture of the firm and its network of partners for creating, marketing, and delivering 


\section{Brasil Business Model - Considerações Sobre o Conceito}

this value and relationship capital, to generate profitable and sustainable revenue streams." (OSTERWALDER et al, 2005, p. 10).

Verstraete e Juisson (2007) apresentam o BM como uma conceptualização dos negócios, envolvendo simultaneamente uma questão concreta - o rendimento (financeiro, ou valor) - e uma questão mais abstrata como se estabelecem as relações dos grupos de interesse para aquele empreendimento particular.

De qualquer forma, a possível explicitação do conceito é difícil visto este estar associado a aspectos dinâmicos do processo empresarial - a criação de valor, a competitividade e a mudança (ARLOTO et al, 2011).

Exemplificando a dificuldade, se forem consideradas os fatores (building blocks) a serem levados em conta na definição de um BM específico, segundo a proposição de Ostenwalder e outros (2005) deverão ser abordados 9 fatores. Já no trabalho de Samir e outros (2012) a discussão se torna ainda mais complexa, dado que na sua pesquisa que envolveu trabalhos de 62 autores nos quais foram identificados 28 fatores comuns.

Às dificuldades citadas, pode ser agregado mais um aspecto - a análise do termo: business + model. Baden-Fuller e Morgan (2010), repetindo o que foi dito por Osterwalder et al (2005) observam que existem ao menos 3 abordagens diferentes sobre o conceito de modelo de um negócio - uma abstração abrangente, que descreveria todos os negócios do mundo; um sistema de classificação que agregaria grupos de empresas que apresentariam características comuns de atuação nos negócios onde operam e, por último, seria a conceptualização de um negócio específico.

No primeiro dos casos, o BM serviria como uma simplificação do processo de decisão e ação empresarial, tratada por Sedon e Lewis (2003) como uma descrição sintética da estratégia efetivamente utilizada pela empresa em um negócio - o meta modelo. Essa sintetização incluiria o cliente alvo, a descrição da oferta feita a esse cliente, a forma como essa oferta seria produzida considerando os fornecedores e a forma pela qual esses grupos de interesse se relacionariam.

Esta questão é dominante na genealogia do conceito - a singularidade do BM e na verdade representa também o terceiro dos casos. Ele é próprio de uma empresa, que atua em um negócio específico (considerado aqui como o relacionamento por meio de transações monetárias entre uma demanda e um conjunto de ofertas), em um ponto do tempo. Sua principal função seria estabelecer uma linguagem comum entre os grupos de interesse - a inserção do BM no contexto da Teoria das Convenções.

No segundo dos casos, conforme Baden-Fuller e Morgan (2010), estariam os BMs dominantes em certos tipos de negócio - as taxonomias de BM's ou sub-meta-modelos de Ostenwalder et al (2005). Esta abordagem comporta simultaneamente problemas e oportunidades. Um dos problemas é que um BM, por ser uma expressão de um comportamento de uma determinada empresa em um determinado negócio, é singular, isto é, não há dois BM's iguais. Além disso, dadas as dinâmicas dos negócios, o BM da empresa dominante hoje pode ser (e provavelmente será) o BM "falido" de amanhã, conforme chamou já observava Rayport no ano de 1999 (RAYPORT, 1999).

No mercado de aviação haveria o "modelo SouthWest" que definiria o negócio de transporte aéreo de passageiros "low-fare, low cost". Mas, o que seria esse "modelo". A SouthWest é uma empresa americana, que só atua nos Estados Unidos continental. Ryanair, a maior companhia europeia de baixa tarifa, bem como a Easy Jet fazem voos internacionais, o que impõe questões tanto de ordem operacional (no mínimo a questão dos idiomas, necessariamente alguma terceirização e sistemas de contabilização locais), quanto financeira (maiores custos em função da internacionalização).

A SouthWest é uma empresa independente, ao contrário das subsidiárias de baixo preço das companhias aéreas tradicionais (HOP! da Air France, Air India Express da Air India) ou ainda outras em situações peculiares como a Aer Lingus, cujos principais acionistas são a Ryanair e o governo irlandês (proprietário de $85 \%$ das ações até 2006) e, em considerando os aspectos de público-alvo, escolheu o viajante que utiliza 0 
meio rodoviário, fato não tão evidente nas outras empresas do mesmo setor. Não parece que o BM da SouthWest seja o BM que define o negócio "low-far, low cost".

A realidade de alguns mercados também prejudica em muito essa idéia, que mistura BM e setor econômico no sub-setor de smartphones do setor de telefonia celular (os aparelhos) convivem competidores que adotam BM's absolutamente diferentes entre si - veja-se, por exemplo, Samsung, Apple, Nokia e agora a Google.

Os poucos pontos em comum são a oferta (o aparelho em si) e alguns segmentos de público-alvo. Os sistemas operacionais são diferentes (Android, para a Samsung e Google, iOS para o iPhone, Windows para a Nokia). Além disso o Android é uma plataforma aberta, enquanto que nos demais são proprietárias. No que tange à forma como a proposta de valor é operacionalizada, as alternativas também são diferentes enquanto que a Samsung e Nokia mantêm parte significativa da manufatura em suas unidades próprias, Google e Apple terceirizam integralmente a produção. Além disso, destas a Google, ao contrário das outras, terceiriza totalmente o projeto, gerenciando especificações e resultados.

0 que se tem nesses casos é um benchmarking - o BM que é referência para a forma de uma empresa atuar em um negócio já definido ou mesmo fazer com que potenciais parceiros - fornecedores de recursos compreendam uma proposição, utilizando uma referência de algum negócio distinto daquele que está sendo proposto de forma mais facilmente inteligível pelos investidores.

Não resta dúvida que entrar no mercado da grande distribuição de alimentos no Brasil, por exemplo, com um baixo nível de risco (estratégico) significa que pode ser escolhido um dos formatos básicos - hipermercados, supermercados, ou lojas de vizinhança, com alto nível de auto-serviço, grande integração com os fornecedores (eventualmente com a terceirização para estes da gerência de categoria), algumas marcas geradoras de tráfego, comunicação de marketing orientada para atrair o cliente para que este entre e compre além do produto promovido (a base da comunicação) e preferencialmente outros, além de um sistema de compras altamente agressivo buscando diminuição do custo da mercadoria vendida que, por seu turno, permitirá trabalhar com preços de venda menores - valor é preço baixo. Isso não impede que as empresas entrem nesse mercado com inovações em seus BM's como o People's Supermarket que aparente segue a receita de baixo risco, mas com uma combinação única de recursos (DATAMONITOR, 2011, LITO, 2012, PEOPLE'S SUPERMARKET, 2013). A função de Recursos Humanos incorpora formalmente a idéia do consumidor como co-produtor - em sendo uma cooperativa, o consumidor literalmente trabalha durante um período de 4 horas mensais. A função de comunicação de Marketing, como habitualmente, busca atrair o consumidor, mas não somente para entrar no supermercado e comprar, mas para lá trabalhar. A integração com os produtores é também muito forte, mas centrada nas idéias de fair trade e desenvolvimento sustentável da economia local.

A função de benchmark dos BM's parece estar sendo utilizada pela RIM (Research in Motion) para revitalizar seu Blackberry. Conforme o Editor da revista online ReadWrite, Dan Riwinski (2013), a RIM certamente deve ter estudado o comportamento de seus concorrentes principais (ou seja, os BM's mais representativos do setor de smartphones) e verificou o que funciona e não funciona, oferecendo facilidades para criação de aplicativos (grande trunfo da oferta da Apple), alterando sua distribuição, diminuindo significativamente os contratos de exclusividade para fornecimento de certos equipamentos para determinadas operadoras (um dos motivos de sucesso da Samsung e de fracasso de certos aparelhos da Nokia e HTC nos EUA).

Essa função do conceito - modelagem no sentido de modelo de referência - pode ser rastreada até as obras de Applegate (2000, 2003 apud SEDON et al, 2004) e Weil e Vitale (2000, apud SEDON et al, 2004), passando mais recentemente por Osterwalder e Pigneur (patronos da sistemática Business Model Canvas) e aqui coloca-se a questão - business model ou business modeling. O BM descreve um conjunto de fatores e eventualmente suas relações no âmbito de uma empresa (específica) numa situação de mercado também específica. Já Business modeling seria um processo (caráter dinâmico do conceito) por meio do qual seriam gerados BM's de amanhã (conforme chamada de capa do livro de Osterwalder e Pigneur). 


\section{Brasil Business Model - Considerações Sobre o Conceito}

\section{Considerações Finais}

A idéia partilhada por muitos na Academia, de que BM é ou pode vir a ser uma ferramenta de análise estratégica e de comportamentos empresariais (VERSTRAETE et al, 2012, LECOQ e DEMIL, 2010, SEDON e LEWIS, 2004) é justificada por Rédis (2009) como decorrente da inadequação das ferramentas existentes frente às mudanças ocorridas na Economia a partir da evolução das TICS, da desregulamentação e da globalização dos mercados, levando ao desmantelamento das cadeias produtivas através das reorganização das atividades e à diluição das fronteiras entre os mercados.

Sedon et al (2004) já propunham que o BM seria a estratégia em um nível de abstração mais elevado do que o normalmente empregado nas discussões sobre estratégia e num nível de detalhe menor, o que é consistente com a idéia de modelo de referência. Citando Weil e

Vitale (2001), os autores observam que estes estão muito mais preocupados com os grupos de interesse (quem) e com os fluxos de informação, produtos e dinheiro (os quês) do que a forma como as empresas se comportarão em função da concorrência (SANDERS et al, 2004).

$\mathrm{O}$ que se pode inferir a partir dos artigos analisados e exemplos apresentados é que BM é um conceito aplicável à empresa em uma situação (negócio) específica, que envolve questões quanto ao comportamento que ela deveria, ou poderia adotar nessa situação e como esse comportamento será operacionalizado buscando a criação e apropriação de valor.

Este foco já foi dado no trabalho seminal de Amit e Zoot (2001) quando eles observam que cada BM é centrado em uma empresa em particular, não obstante seu escopo (como unidade de análise) ser mais amplo que a própria empresa, envolvendo, segundo eles, questões relativas às cadeias de valor, inovação (sob a ótica Shumpeteriana), visão baseada em recursos e custos de transação.

De toda forma, aparentemente o conceito de BM já está se tornando, por um lado, uma poderosa ferramenta para uso dos praticantes, deixando para trás seu uso restrito à área de $\mathrm{TI}$, atingindo setores tradicionais, como no exemplo do People's Supermarket apresentado e, por outro, mais uma ferramenta de análise de negócios a ser utilizada pelos acadêmicos, superando algumas dificuldades decorrentes da radical modificação dos comportamentos das organizações nas suas ações de mercado. Vale dizer que o BM é uma ferramenta importantíssima para os empreendedores - para Amit e Zoot (2001) BM é elaborado com base nos referenciais teóricos oriundos das áreas de administração estratégica e empreendedorismo considerados de forma integrada, não como uma soma de suas contribuições.

Em termos práticos, ela permite adequar o discurso dos empreendedores às convenções aceitáveis por diferentes grupos de interesse, facilitando o processo de transformação dos fornecedores de recursos em grupos de interesse. Permite também definir e compreender as linhas gerais de atuação/comportamento da empresa em um determinado negócio.

Este conceito, tal como outros no passado recente (Balance Scorecard, por exemplo), aproxima os acadêmicos dos praticantes - vale lembrar que a proposta de Ostenwalder e Pigneur, Business Model Canvas foi elaborada a partir do levantamento das práticas empresariais.

\section{Notas}

${ }^{1}$ A título de ilustração, em uma pesquisa realizada em 13/04/13, por meio do mecanismo de busca Google com a expressão "business model", restringindo os resultados em língua portuguesa, foram obtidos aproximadamente 192.000 resultados.

1 Aplicou-se aqui o termo "noção" em conformidade com o Merrian -Webster: 1) uma impressão ou concepção de algo conhecido, experienciado ou imaginado, [...]3) uma teoria ou crença adotada por uma pessoa ou grupo . 
1 Em um e-business, no original. Seguindo a percepção de muitos dos autores aqui citados, o constructo de BM para os e-business é o mesmo utilizado para negócios fora da rede.

1 No entanto, não foi usada a expressão exata - "Several of them [kinds of research] have been carried on since the early days of insurance in this country; some have won world-wide renown and, as techniques, have served as models for other types of business." (LANG, 1947, p. 66, não destacado no original)

1 Pesquisa realizada em 12/04/2013

1 Um dos textos veio referenciado duas vezes, vale dizer que dos 17 restantes, 2 eram editoriais e um deles um texto introdutório a uma edição especial.

${ }^{1}$ No trabalho de Ostenwalder et al (2005) esta última forma de expressão é denominada meta-modelo.

1 Esta pergunta hipotética foi criada com base nas proposições de Chesbrough (2003)

1 Esta seria uma possível hipótese a ser testada, considerando as características geralmente apresentadas por um BM que são apresentadas ao longo do texto.

1 Grande número de artigos elabora listas e quadros de conceitos sobre BM, como por exemplo, Joia e Ferreira, 2005, Sabir et al, 2012. A escolha deste artigo em particular deveu-se ao fato de ter sido escrito por ser um dos autores, dentre os pesquisados, mais profícuos em termos de publicações sobre o conceito e pelo artigo estar em um número especial do periódico no qual diversas conceituações do termo foram apresentadas

1 Os autores utilizam o termo resources (recursos). No entanto, pequenas variações de produto, como por exemplo, cor de um automóvel, aproveitando aquele oferecido no artigo, necessita de novas tintas - um recurso - mas que não significa, necessariamente, um novo BM.

\section{Referências Bibliográficas}

AMIT, Rafael. ZOTT, Christoph. Value Creation in e-Business. Strategic Management Journal, 22, 2001 pp. 493-520

ANDREW Parker. Aer Lingus urges shareholders take no action. Financial Times, 20/06/2012. disponível em http://www.ft.com/cms/s/0/06463258-ba36-11e1-84dc-00144feabdc0.html\#axzz2Vter0Dkz, acesso em 11/06/2013.

ARLOTTO, Jaques, SAHUT, Jean-Michel, TEULON, Frédéric. Le Concept de Business Model au travers de la Literature. Gestion 2000. Julliet-Août, 2011. pp.33-47

BADEN-FULLER, Charles, MORGAN, Mary S. Business Model as Model. Long Range Planning. Vol. 43 (2/3), 2010. pp. 156-171

CASADESUS-MASANEL, Ramon, RICART, Joan Enric. From Strategy to Business Models onto Tatics. Long Range Planning. Vol. 43 (2/3), 2010. pp. 195-215

CHARTRAND, Suzane-G., De KONICK, Godelieve. La Clarté Terminologique pour plus de Cohérence et Rigueur dans l'Enseigment du Français (suite). Québec Français. Vol 154, Été, 2009. pp. 143-145.

CHESBROUGH, Henry, ROSEMBLOOM, Richard S. The Role of Business Model in Capturing Value from Innovation: evidences from Xerox Corportation's technology spin-off companies. Industrial and Corporate Change. Vol 11(3), 2002. pp. 529-555

CHESBROUGH, Henry. Open Innovation: The New Imperative for Creating and Profiting from Technology. Boston: Harvard Business School Press. 2003

DATAMONITOR. The People's Supermarket Case Study. 2011. Disponível na Base de Dados EBSCOHost, Business Source Complet. 


\section{Brasil Business Model - Considerações Sobre o Conceito}

DEMIL, Benoît, LECOCQ, Xavier, VARNIER, Vanessa. Le Business Model: l'oublié de la stratégie. 13e Conférence de l'Aims. Normandie. Vallée de Seine, 2, 3 et 4 juin 2004.

ETHIRAJ, Sendil, GULER, Isin, SINGH, Arbir. The Impact of Internet and Electronic Technologies on Firms and its Implications for Competitive Advantadge. Disponível em http://knowledge.emory.edu/papers/977.pdf, acesso, 21/02/2013.

HEDMAN, Jonas, KALLING, Thomas. The Business Model: a means to comprehend the management and business context of information and comunication technology. European Conference on Information Systems. Gdansk, Polônia, 6,7,8 Juin, 2002. pp. 148-162.

JOIA, Luiz Antônio, FERREIRA, Sinval. Modelo de Negócios: constructo real ou metáfora de estratégia. Cadernos EBAPE.BR

JONES, Gardner N. Educators, Electrons and Business Models: a problem in synthesis. The Accounting Review. October, 1960. pp. 619-626.

JONHSON, Mark W., CHRISTENSEN, Clayton M. KAGERMANN, Henning. Reinventing your Business Model. Harvard Business Review. Vol (86) Dec., 2008. pp. 50-59

LANG, Frank. Insurance Research. The Journal of Marketing. July, 1947. pp. 66-71.

LITO, Guilherme. O Supermercado mais Inovador do Mundo. Blog Luz.vc, 29/11/2011. Disponível em http://blog.luz.vc/inovacao/o-supermercado-mais-inovador-do-mundo/, acesso em 11/06/2013.

MAGRETA, Joan. Why Business Model Matter. Harvard Business Review. May, 2002. pp.86-92.

MARTINS, Gilberto de Andrade. Sobre Conceitos, Definições e Constructos nas Ciências Administrativas. Gestão e Regionalidade. Ano 22, № 62, Jul-Dez, 2005. pp. 28-35

MERRIAN -WEBSTER DICTIONARY, versão on-line, disponivel em http://www.merriamwebster.com/dictionary/notion, acesso em 14/04/2013

MORRIS, Michael, SHINDEHUTTE, Minet, ALLEN, Jeffrey. The Entrepreneur's Business Model: toward a unified perspective. Journal of Business Research. Vol 58(6), 2005. pp. 726-735

OSTERWALDER, Alexander, PIGNEUR, Yves, TUCCI, Christopher L. Claryfing Business Model: origins, present and future of the concept. Communications of the Association of Infomation Systems. Vol 16, 2005. pp. $1-25$

PATELI, Adamantia White Paper: A Domain Area Report on Business Model. Athens University of Economics and Business, Department of Science \& Technology; Eltrun, The eBusiness Center. Athens, 20/11/2002, Version 1.3

PEOPLE'S SUPERMARKET. About Us. 2013. Disponível em http://www.thepeoplessupermarket.org/aboutus/, acesso em 11/06/2013.

RAYPORT, Jefrey. F. 1999. The truth about Internet business models. Strategy+Business, Third Quarter(16), 1-3.1999, disponível em http://www.strategy-business.com/article/19334?gko=6518d, acesso em 11/06/2013

RIWINSKI, Dan. RIM's BlackBerry 10 Distribution Strategy Looks Very Smart. ReadWright, 10/01/2013. Disponivel em http://readwrite.com/2013/01/10/rims-blackberry-10-distribution-strategy-looks-verysmart\#awesm= 08soaCVIW1KUqb, acesso em 16/06/2013

SABIR, M.Suleman et al. Theoretical Foundation of Business Model and their Building Blocks. Journal of Management Research. Vol 4 (4), 2012. pp.160-179. 
SEDDON, Peter B. , LEWIS, Geoffrey, FREEMAN, Phil, SHANKS, Graeme. The Case for Viewing Business Models as Abstractions of Strategy. Communications of the Association of Infomation Systems. Vol 13. 2004. pp. 427-442

SEDDON, Peter B. , LEWIS, Geoffrey. Strategy and Business Models: what's the difference? 7th Pacific Asia Conference on Information Systems, 10-13 July 2003, Adelaide, South Australia. pp. 236-248.

STANFORD, Richard A. Ceteris Paribus Methodology and Computerized Economic-Business Models. The Journal of Economic Education. Fall, 1972. pp. 23-29

TEECE, David J. Business Models, Business Strategy and Innovation. Long Range Planning. Vol.43 (2/3), 2010. pp. $172-194$

TIMMERS, Paul. Business Models for Electronic Markets. Electronic Markets. Vol 8 (2), 1998. pp. 3-8.

VERSTRAETE, Thierry, JOUISSON Estèle. Trois Théories pour Conceptualizer la Notion de Business Model em Contexte de Création d'Entreprise. XVlème Conférence de Management Stratégique, Montrèal, 6-9 Juin 2007. pp. 1-33 Vol III (4), Dez. 2005. pp. 1-18

ZOTT, Christoph, AMIT, Rafael, MASSA, Lorenzo. The Business Model; theoretical roots, recent developments, and future research. IESE (University of Navarra), Working Paper WP - 862. June, 2010, revised september 2010. pp. 1-45 